\title{
CLOTHOID SPLINE TRANSITION SPIRALS
}

\author{
D. S. MEEK AND D. J. WALTON
}

\begin{abstract}
Highway and railway designers use clothoid splines (planar $G^{2}$ curves consisting of straight line segments, circular arcs, and clothoid segments) as center lines in route location. This paper considers the problem of finding a clothoid spline transition spiral which joins two given points and matches given curvatures and unit tangents at the two points. Conditions are given for the existence and uniqueness of the clothoid spline transition spirals, and algorithms for finding them are outlined.
\end{abstract}

\section{INTRODUCTION}

The Fresnel integrals [1, p. 300],

$$
C(t)=\int_{0}^{t} \cos \frac{\pi}{2} u^{2} d u \text { and } S(t)=\int_{0}^{t} \sin \frac{\pi}{2} u^{2} d u,
$$

are nonnegative functions of $t, t \geq 0$. The integrals of the Fresnel integrals,

$$
C_{I}(t)=\int_{0}^{t} C(u) d u=t C(t)-\frac{1}{\pi} \sin \frac{\pi}{2} t^{2}
$$

and

$$
S_{I}(t)=\int_{0}^{t} S(u) d u=t S(t)+\frac{1}{\pi} \cos \frac{\pi}{2} t^{2}-\frac{1}{\pi}
$$

are nonnegative, strictly monotone increasing functions of $t, t \geq 0$. A recent paper [7] gives convenient rational approximations to the Fresnel integrals. With the above formulae, those approximations also give convenient approximations to the integrals of the Fresnel integrals.

The clothoid is a spiral which can be defined in terms of Fresnel integrals as

$$
\pi B\left(\begin{array}{l}
C(t) \\
S(t)
\end{array}\right)
$$

where the scaling factor $\pi B$ is positive and the parameter $t$ is nonnegative.

Received by the editor May 20, 1990 and, in revised form, January 28, 1991.

1991 Mathematics Subject Classification. Primary 65D05.

This research was supported by grants from the Natural Sciences and Engineering Research Council of Canada, and one of the authors acknowledges the hospitality of the University of Victoria while on sabbatical. 


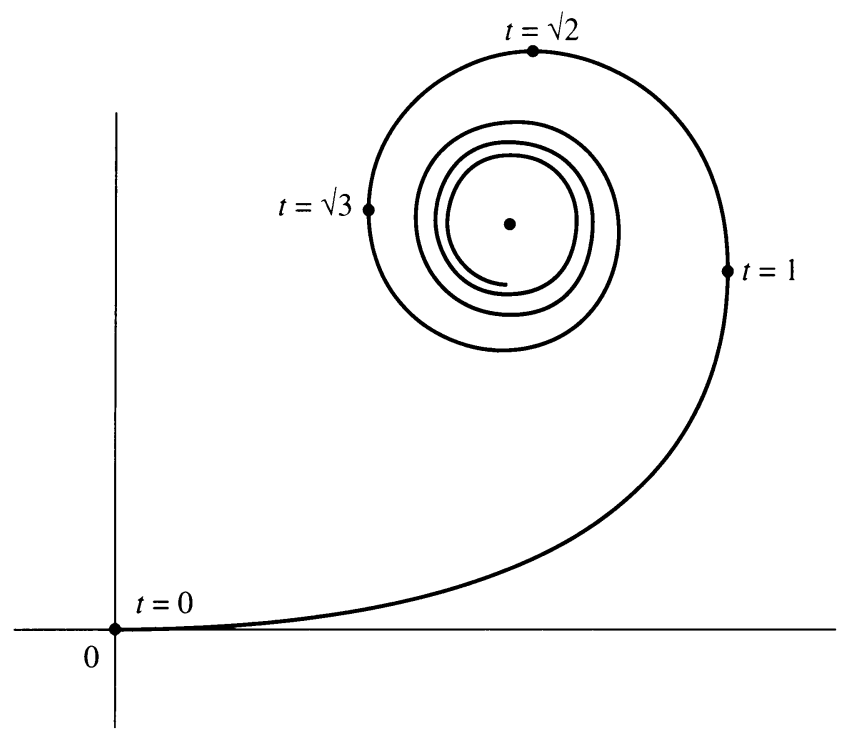

FIGURE 1. Part of a clothoid

The clothoid (2) is in the first quadrant, starts at the origin with $t=0$, and approaches the limiting point $\left(\frac{\pi}{2} B, \frac{\pi}{2} B\right)$ as $t$ approaches infinity (see Figure 1). Some useful formulae for the clothoid follow: the angle that the tangent forms with the $X$-axis is $\frac{\pi}{2} t^{2}$, the curvature is $\frac{t}{B}$, an element of arc length is $d s=\pi B d t$, and the center of the circle of curvature is

$$
\frac{\pi B}{t}\left(\begin{array}{c}
C_{I}(t) \\
S_{I}(t)+\frac{1}{\pi}
\end{array}\right) .
$$

A planar $G^{2}$ curve (a curve which is twice continuously differentiable with respect to arc length [4, p. 151]) consisting of clothoid segments, circular arcs, and straight line segments is called a clothoid spline, as circles and straight lines can be considered limiting forms of clothoids. Clothoid splines are used in the route design of the center lines of highways and railways [2]. Route designers think of the clothoid segments in clothoid splines as transitional curves between straight lines and circular arcs, and between circular arcs of different radii. Clothoid splines have been studied recently in [10,11, 12]. A clothoid spline is expressed parametrically, and has the attractive feature that the arc length and curvature are continuous piecewise linear functions of the parameter.

Algorithms for finding clothoid segments to join given circles and straight lines, and produce a $G^{2}$ curve, have been described in [9, 13]. The result is a clothoid spline, but it is not possible to specify the points on the given circles or straight lines where the clothoid segment starts or ends. Algorithms for joining a point on a circle or straight line and another point with a clothoid segment so that the circle or straight line and clothoid segment form a planar $G^{2}$ curve have been described in [8]. However, in that paper, it is not possible to specify the curvature and the unit tangent at the second point beforehand. In this paper, a clothoid spline transition spiral is found to join two given points, and match curvatures and unit tangents at the two points. This is a type of Hermite interpolation, and will henceforth be called a Hermite matching. 


\section{TERMINOLOGY}

Much of the terminology required in the paper is established in this section. A tangent to a curve is considered to be a vector which points in the direction of increasing arc length. The curvature is positive (negative) if the center of curvature is on the left (right) when moving in a direction of increasing arc length. A spiral is a curve whose curvature is monotone increasing or monotone decreasing and does not change sign.

The interpolation problem to be solved is to find a clothoid spline spiral which joins $\mathbf{P}$ to $\mathbf{Q}$, and which has given unit tangents $\mathbf{t}_{\mathbf{P}}, \mathbf{t}_{\mathbf{Q}}$, and given curvatures $K_{\mathbf{P}}, K_{\mathbf{Q}}$, at $\mathbf{P}$ and $\mathbf{Q}$ (Hermite matching). It may be assumed, without loss of generality, that the curvature of the clothoid spline spiral is nonnegative and nondecreasing. For an interpolation problem with $K_{\mathbf{P}}=K_{\mathbf{Q}}$, the clothoid spline spiral would have constant curvature and would be a circular arc or straight line. It is easy to determine if it is possible to match the given data with a circular arc or straight line. Henceforth, assume that $0 \leq K_{\mathbf{P}}<K_{\mathbf{Q}}$.

A standard form of the above interpolation problem is obtained by placing $\mathbf{P}$ at the origin, and aligning $t_{p}$ along the positive $X$-axis (see Figure 2). Assume that the tangent to the clothoid spline spiral rotates through an angle less than $\pi$ when traversing the curve from $\mathbf{P}$ to $\mathbf{Q}$. This is not a severe restriction, and it is made to facilitate proofs of the lemmas below. Let the angle the tangent $\mathbf{t}_{\mathbf{Q}}$ forms with the $X$-axis be $\frac{\pi}{2} W, 0<W<2$.

For some choices of $\mathbf{Q}, W, K_{\mathbf{P}}$, and $K_{\mathbf{Q}}$, there is no spiral of nonnegative, nondecreasing curvature joining the origin $\mathbf{P}$ to $\mathbf{Q}$ with a Hermite matching. For example, it is impossible to find such a spiral if $\mathbf{Q}$ is below the $X$-axis.

Definition. Let the region $\Gamma=\Gamma\left(\mathbf{P}, W, K_{\mathbf{P}}, K_{\mathbf{Q}}\right)$ be the set of points $\mathbf{Q}$ such that there exists a spiral of nonnegative, nondecreasing curvature which connects $\mathbf{P}$ to $\mathbf{Q}$ with a Hermite matching.

With the point $\mathbf{R}$ defined as

$$
\mathbf{R}=\frac{1}{K_{\mathbf{Q}}}\left(\begin{array}{c}
\sin \frac{\pi}{2} W \\
1-\cos \frac{\pi}{2} W
\end{array}\right),
$$

the following theorem describes the region $\Gamma$ for $K_{\mathbf{P}}=0$ and for $K_{\mathbf{P}}>0$.

Theorem 1. If $K_{\mathbf{P}}=0$, the region $\Gamma$ is the interior of the wedge bounded by the ray from $\mathbf{R}$ making an angle of $\frac{\pi}{4} W$ with the $X$-axis and the ray from $\mathbf{R}$

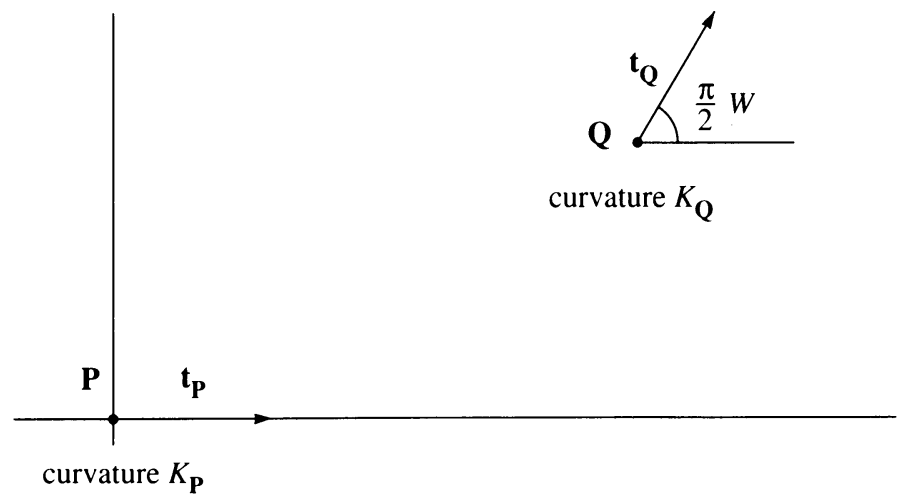

FIGURE 2. The interpolation problem in standard form 


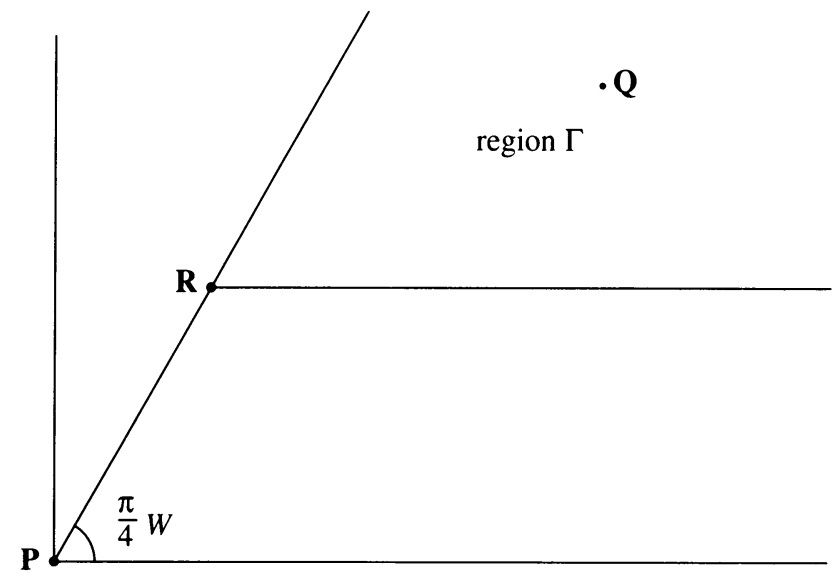

Figure 3. The region $\Gamma$ when $K_{\mathbf{P}}=0$

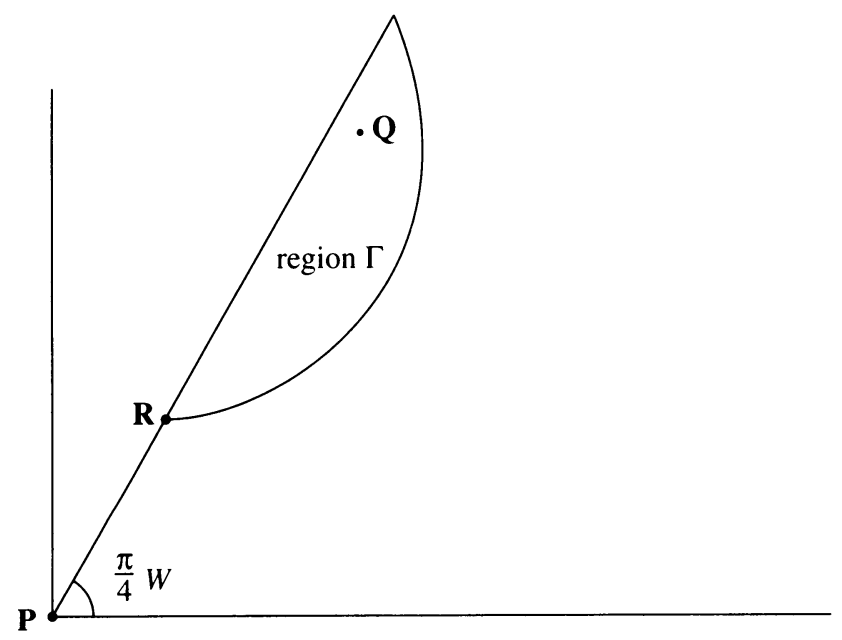

FIGURE 4. The region $\Gamma$ when $K_{\mathbf{P}}>0$

parallel to the $X$-axis (see Figure 3 ). If $K_{\mathbf{P}}>0$, the region $\Gamma$ is the segment of the circle (3) which is to the right of the ray from $\mathbf{R}$ making an angle of $\frac{\pi}{4} W$ with the $X$-axis (see Figure 4).

Proof. The region $\Gamma$ can be found using theorems in Guggenheimer [6]. Vogt's theorem [6, p. 49] states that the angle $t_{\mathbf{Q}}$ makes with $\mathbf{P Q}$ is greater than the angle $\mathbf{P Q}$ makes with the $X$-axis. This means that $\mathbf{Q}$ must be to the right of the straight line through the origin which makes an angle $\frac{\pi}{4} W$ with the $X$-axis.

Kneser's theorem [6, p. 48] states that any circle of curvature of a spiral contains every smaller circle of curvature in its interior. For $K_{\mathbf{P}}=0$ and a spiral of nonnegative curvature, this can be interpreted to mean that the circle of curvature at $\mathbf{Q}$ must be entirely above the $X$-axis. Thus, the $Y$-component of $\mathbf{Q}$ must be greater than $\left(1-\cos \frac{\pi}{2} W\right) / K_{\mathbf{Q}}$. For $K_{\mathbf{P}}>0$, the condition that the circle of curvature at $\mathbf{P}$ must entirely enclose the circle of curvature at $\mathbf{Q}$ can be replaced by the condition that the distance between centers of those two circles of curvature is less than the positive difference of their radii. This leads 
to the fact that $\mathbf{Q}$ must be in the following circle:

$$
\text { center : }\left(\begin{array}{c}
\frac{1}{K_{\mathbf{Q}}} \sin \frac{\pi}{2} W \\
\frac{1}{K_{\mathbf{P}}}-\frac{1}{K_{\mathbf{Q}}} \cos \frac{\pi}{2} W
\end{array}\right), \quad \text { radius : } \frac{1}{K_{\mathbf{P}}}-\frac{1}{K_{\mathbf{Q}}} \text {. }
$$

Guggenheimer [6, p. 52] shows that the conditions in Vogt's and Kneser's theorems are also sufficient conditions on $\mathbf{Q}$ that a spiral can be found to join $\mathbf{P}$ to $\mathbf{Q}$, given $W, K_{\mathbf{P}}, K_{\mathbf{Q}}$.

The main mathematical result of this paper is that for any $\mathbf{Q}$ in $\Gamma$, there is a unique clothoid spline spiral (of type A or type B, cf. $\S \S 4$ and 5, respectively) joining $\mathbf{P}$ to $\mathbf{Q}$ with a Hermite matching. This result will be proven in $\S 6$.

\section{Partition OF THE REgION $\Gamma$}

The region $\Gamma$ is partitioned into two subregions $\Gamma_{\mathrm{A}}$ and $\Gamma_{\mathrm{B}}$ by the two curves $\mathbf{D}(t)$ and $\mathbf{E}(t)$ (see (6a), (6b), (7), and (8)) because the type of clothoid spline spiral which will be used depends on where $\mathbf{Q}$ is in $\Gamma$. If $\mathbf{Q}$ is at $\mathbf{S}$, the meeting point of $\mathbf{D}(t)$ and $\mathbf{E}(t)$, a single clothoid segment can be used to give a Hermite matching. If $\mathbf{Q}$ is on $\mathbf{D}(t)$, a clothoid spline spiral comprising a clothoid segment and a circular arc of angle $\frac{\pi}{2} t$ can be used to give a Hermite matching. If $\mathbf{Q}$ is on $\mathbf{E}(t)$, a clothoid spline spiral comprising a line of length $t$ or a circular arc of angle $\frac{\pi}{2} t$, and a clothoid segment can be used to give a Hermite matching. In $\S 4$, it is shown that a clothoid spline spiral comprising a line or circular arc, a clothoid segment, and another circular arc, and matching $W, K_{\mathbf{P}}$, and $K_{\mathbf{Q}}$ can reach any point in $\Gamma_{\mathbf{A}}$ uniquely. In $\S 5$, it is shown that a clothoid spline spiral comprising a clothoid segment, a circular arc, and another clothoid segment, and matching $W, K_{\mathbf{P}}$, and $K_{\mathbf{Q}}$ can reach any point in $\Gamma_{\mathbf{B}}$ uniquely.

Define the matrix of rotation by $\frac{\pi}{2} \omega$,

$$
R(\omega)=\left(\begin{array}{cc}
\cos \frac{\pi}{2} \omega & -\sin \frac{\pi}{2} \omega \\
\sin \frac{\pi}{2} \omega & \cos \frac{\pi}{2} \omega
\end{array}\right),
$$

and the variable $B$ for formulae in this section,

$$
B=\sqrt{\frac{W-t}{K_{\mathbf{Q}}^{2}-K_{\mathbf{P}}^{2}}} .
$$

The curve $\mathbf{D}(t)$ for $0 \leq t \leq W$ is

$$
\begin{aligned}
\mathbf{D}(t)= & \pi B R\left(-K_{\mathbf{P}}^{2} B^{2}\right)\left(\begin{array}{c}
C\left(K_{\mathbf{Q}} B\right)-C\left(K_{\mathbf{P}} B\right) \\
S\left(K_{\mathbf{Q}} B\right)-S\left(K_{\mathbf{P}} B\right)
\end{array}\right) \\
& -\frac{1}{K_{\mathbf{Q}}}\left(\begin{array}{c}
-\sin \frac{\pi}{2} W+\sin \frac{\pi}{2}(W-t) \\
\cos \frac{\pi}{2} W-\cos \frac{\pi}{2}(W-t)
\end{array}\right),
\end{aligned}
$$

and the point $\mathbf{S}=\mathbf{D}(0)$. When $K_{\mathbf{P}}=0, \mathbf{D}(t)$ can be written more compactly as

$$
\mathbf{D}(t)=\frac{\pi}{K_{\mathbf{Q}}}\left(\begin{array}{l}
C_{I}(\sqrt{W-t}) \\
S_{I}(\sqrt{W-t})
\end{array}\right)+\mathbf{R} .
$$

Note that in both cases $\mathbf{D}(W)=\mathbf{R}$. When $K_{\mathbf{P}}=0$ and $t \geq 0$, the curve $\mathbf{E}(t)$ is

$$
\mathbf{E}(t)=\mathbf{S}+\left(\begin{array}{l}
t \\
0
\end{array}\right),
$$




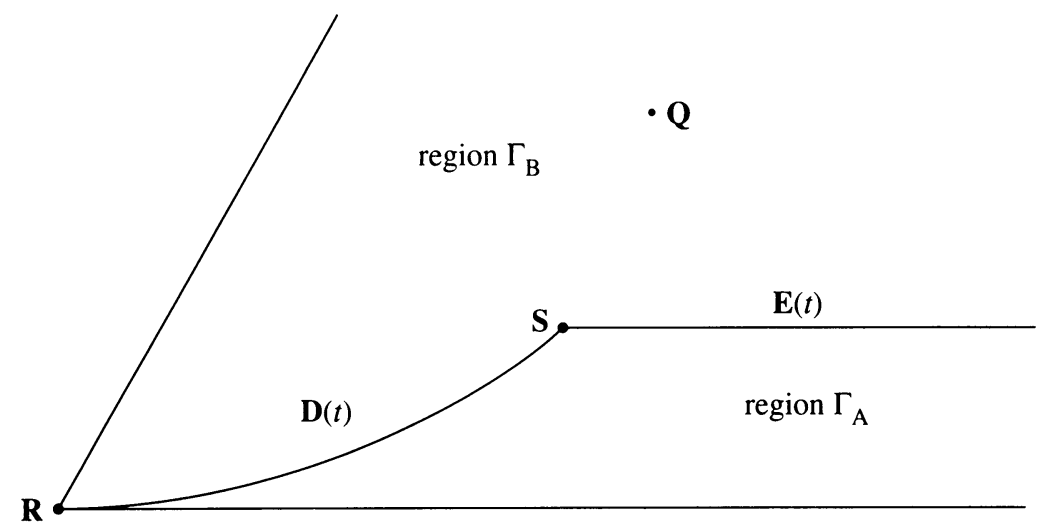

FIGURE 5. The partition of $\Gamma$ when $K_{\mathbf{P}}=0$

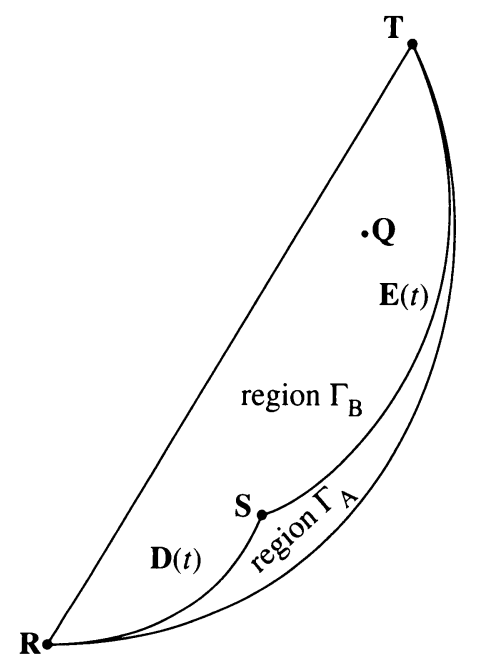

FIGURE 6. The partition of $\Gamma$ when $K_{\mathbf{P}}>0$

and, when $K_{\mathbf{P}}>0$ and $0 \leq t \leq W$, the curve $\mathbf{E}(t)$ is

$$
\mathbf{E}(t)=\pi B R\left(t-K_{\mathbf{P}}^{2} B^{2}\right)\left(\begin{array}{c}
C\left(K_{\mathbf{Q}} B\right)-C\left(K_{\mathbf{P}} B\right) \\
S\left(K_{\mathbf{Q}} B\right)-S\left(K_{\mathbf{P}} B\right)
\end{array}\right)+\frac{1}{K_{\mathbf{P}}}\left(\begin{array}{c}
\sin \frac{\pi}{2} t \\
1-\cos \frac{\pi}{2} t
\end{array}\right) .
$$

Note that in both cases $\mathbf{E}(0)=\mathbf{S}$.

The region $\Gamma$ is divided into two disjoint subregions, $\Gamma_{\mathrm{A}}$ and $\Gamma_{\mathrm{B}}$, by the curve $\mathbf{D}(t)$ which runs from $\mathbf{R}$ to $\mathbf{S}$, and by $\mathbf{E}(t)$, which is a straight line from $\mathbf{S}$ parallel to the $X$-axis when $K_{\mathbf{P}}=0$, and which is a convex curve from $\mathbf{S}$ to $\mathbf{T}=\mathbf{E}(W)$ when $K_{\mathbf{P}}>0$ (see Figures 5 and 6 ).

If it is possible to join $\mathbf{P}$ to $\mathbf{Q}$ with a spiral, then $\mathbf{Q}$ is in $\Gamma$. Since the boundaries of $\Gamma$ are straight lines and circular arcs, it is easy to test a given point to see if it is in $\Gamma$. However, the goal is to join $\mathbf{P}$ to $\mathbf{Q}$ with a clothoid spline spiral. The type of clothoid spline spiral to be used depends on whether $\mathbf{Q}$ is in $\Gamma_{\mathrm{A}}, \Gamma_{\mathrm{B}}$ or on the boundary between those two regions.

Algorithm 1. How to determine whether $\mathbf{Q}$ is in $\Gamma_{A}, \Gamma_{B}$ or on the boundary between those two regions. 
Lemmas 1 and 2 show that $\mathbf{D}(t)$ and $\mathbf{E}(t)$ are curves whose curvature is positive, and whose tangents rotate through an angle of less than $\pi$. These are the conditions required by the algorithm WhichSide [8] which can determine whether a given point is on, on the convex side of, or on the concave side of a parametric curve. When $\mathbf{Q}$ is in $\Gamma$ but is not on either of the curves $\mathbf{D}(t)$ and $\mathbf{E}(t)$, it must be determined whether $\mathbf{Q}$ is in $\Gamma_{\mathrm{A}}$ or in $\Gamma_{\mathrm{B}}$. If $\mathbf{Q}$ is on the convex side of $\mathbf{D}(t)$, and below $\mathbf{E}(t)$ when $K_{\mathbf{P}}=0$, or if $\mathbf{Q}$ is on the convex side of $\mathbf{D}(t)$ and on the convex side of $\mathbf{E}(t)$ when $K_{\mathbf{P}}>0$, then $\mathbf{Q}$ is in $\Gamma_{\mathbf{A}}$, otherwise $\mathbf{Q}$ is in $\Gamma_{\mathrm{B}}$.

Lemma 1. The curve $\mathbf{D}(t),(5)$ and (6a), from $\mathbf{R}$ to $\mathbf{S}$, is a curve of positive curvature and the tangent rotates through an angle of less than $\pi$.

Proof. The curvature of $\mathbf{D}(t)$ running from $\mathbf{S}$ to $\mathbf{R}$ is

$$
\frac{\operatorname{det}\left(\mathbf{D}^{\prime}(t), \mathbf{D}^{\prime \prime}(t)\right)}{|\mathbf{D}(t)|^{3}}
$$

where the sign of the numerator determines the sign of the curvature. The function $\mathbf{D}(t)$, written with integrals, is

$$
\mathbf{D}(t)=\pi B \int_{K_{\mathbf{P}} B}^{K_{\mathbf{Q}} B}\left(\begin{array}{c}
\cos \frac{\pi}{2}\left(u^{2}-K_{\mathbf{P}}^{2} B^{2}\right) \\
\sin \frac{\pi}{2}\left(u^{2}-K_{\mathbf{P}}^{2} B^{2}\right)
\end{array}\right) d u-\frac{1}{K_{\mathbf{Q}}}\left(\begin{array}{c}
-\sin \frac{\pi}{2} W+\sin \frac{\pi}{2}(W-t) \\
\cos \frac{\pi}{2} W-\cos \frac{\pi}{2}(W-t)
\end{array}\right) .
$$

The substitution $U=u^{2}-K_{\mathbf{P}}^{2} B^{2}$ transforms the integral term in the above into

$$
\frac{\pi}{2} \int_{0}^{W-t} \frac{B}{\sqrt{U+K_{\mathbf{P}}^{2} B^{2}}}\left(\begin{array}{c}
\cos \frac{\pi}{2} U \\
\sin \frac{\pi}{2} U
\end{array}\right) d U
$$

Differentiating with respect to $t$, we get

$$
\mathbf{D}^{\prime}(t)=\frac{-\pi}{4\left(K_{\mathbf{Q}}^{2}-K_{\mathbf{P}}^{2}\right)} \int_{0}^{W-t} \frac{u}{B\left(u+K_{\mathbf{P}}^{2} B^{2}\right)^{3 / 2}}\left(\begin{array}{c}
\cos \frac{\pi}{2} u \\
\sin \frac{\pi}{2} u
\end{array}\right) d u
$$

and

$$
\begin{aligned}
\mathbf{D}^{\prime \prime}(t)= & \frac{\pi}{4 K_{\mathbf{Q}}^{3} B^{2}}\left(\begin{array}{c}
\cos \frac{\pi}{2}(W-t) \\
\sin \frac{\pi}{2}(W-t)
\end{array}\right) \\
& -\frac{\pi B}{8(W-t)^{2}} \int_{0}^{W-t} \frac{u\left(u+4 K_{\mathbf{P}}^{2} B^{2}\right)}{\left(u+K_{\mathbf{P}}^{2} B^{2}\right)^{5 / 2}}\left(\begin{array}{c}
\cos \frac{\pi}{2} u \\
\sin \frac{\pi}{2} u
\end{array}\right) d u .
\end{aligned}
$$

The numerator of the curvature, $\operatorname{det}\left(\mathbf{D}^{\prime}(t), \mathbf{D}^{\prime \prime}(t)\right)$, consists of two terms. The first term is a negative multiple of

$$
\int_{0}^{W-t} \frac{u}{\left(u+K_{\mathbf{P}}^{2} B^{2}\right)^{3 / 2}} \sin \frac{\pi}{2}(W-t-u) d u
$$

where the integral is positive since $W-t \leq W<2$, and the second term is a positive multiple of

$$
\int_{0}^{W-t} \int_{0}^{W-t} \frac{u}{\left(u+K_{\mathbf{P}}^{2} B^{2}\right)^{3 / 2}} \frac{v\left(v+4 K_{\mathbf{P}}^{2} B^{2}\right)}{\left(v+K_{\mathbf{P}}^{2} B^{2}\right)^{5 / 2}} \sin \frac{\pi}{2}(v-u) d u d v
$$

The identity

$$
\int_{a}^{b} \int_{a}^{b} f(u, v) d u d v=\int_{a}^{b} \int_{a}^{v}\{f(u, v)+f(v, u)\} d u d v
$$


applied to the above double integral, transforms it into a negative multiple of

$$
\int_{0}^{W-t} \int_{0}^{v} \frac{u v(v-u)}{\left(u+K_{\mathbf{P}}^{2} B^{2}\right)^{5 / 2}\left(v+K_{\mathbf{P}}^{2} B^{2}\right)^{5 / 2}} \sin \frac{\pi}{2}(v-u) d u d v .
$$

This double integral is positive since $W-t \leq W<2$. Both terms in $\operatorname{det}\left(\mathbf{D}^{\prime}(t), \mathbf{D}^{\prime \prime}(t)\right)$ are negative; so the curvature of $\mathbf{D}(t)$ from $\mathbf{S}$ to $\mathbf{R}$ is negative. Thus, the curvature of $\mathbf{D}(t)$ from $\mathbf{R}$ to $\mathbf{S}$ is positive.

The value of $\mathbf{D}^{\prime}(0)$ from (9) is a positive multiple of

$$
-\int_{0}^{W} \frac{u}{\left(u+K_{\mathbf{P}}^{2} B^{2}\right)^{3 / 2}}\left(\begin{array}{c}
\cos \frac{\pi}{2} u \\
\sin \frac{\pi}{2} u
\end{array}\right) d u .
$$

This vector points into the third or fourth quadrant. Differentiating (6a) directly and setting $t=W$, which means $B=0$, the value of $\mathbf{D}^{\prime}(W)$ is a positive multiple of $\left(\begin{array}{c}-1 \\ 0\end{array}\right)$. The tangent to the curve $\mathbf{D}(t)$ when traversing the curve from $\mathbf{R}$ to $\mathbf{S}$ points in the opposite direction to the above vectors and thus rotates through an angle of less than $\pi$.

Lemma 2. When $K_{\mathbf{P}}>0$, the curve $\mathbf{E}(t),(5),(8)$, from $\mathbf{S}$ to $\mathbf{T}$ is a curve of positive curvature and the tangent rotates through an angle of less than $\pi$.

Proof. The proof is very similar to the proof of Lemma 1 and will be omitted.

\section{Clothoid SPLine SPIRAL OF TYPE A}

In this section, a clothoid spline spiral which can reach any point in $\Gamma_{\mathrm{A}}$ will be described. The proof that the clothoid spline spiral is unique for any point in $\Gamma_{A}$, and a short description of an algorithm for finding the clothoid spline spiral, will be given.

With $K_{\mathbf{P}}=0$, a clothoid spline spiral of type A consists of a straight line followed by a clothoid segment which is followed by a circular arc (see Figure 7). Let the length of the straight line be $x$, let the scaling factor of the clothoid segment be $\pi B$, and let the angle of the circular arc be $\frac{\pi}{2} L$. To match curvatures, the parameter in the clothoid segment must run from $t=0$ to $t=K_{\mathbf{Q}} B$. The total angle through which the tangent rotates when traversing the curve from $\mathbf{P}$ to $\mathbf{Q}, \frac{\pi}{2} W$, must equal the sum of the angles through which the tangent rotates over the clothoid segment and over the circular arc. Equating angles gives

$$
W=K_{\mathbf{Q}}^{2} B^{2}+L
$$

from which $B$ is found to be $B=\sqrt{W-L} / K_{\mathbf{Q}}$. The circular arc is part of the circle of curvature of the clothoid at $t=K_{\mathbf{Q}} B$. A formula for $\mathbf{Q}$ is the center of that circle of curvature plus a radius vector which is perpendicular to $\mathbf{t}_{\mathbf{Q}}$,

$$
\mathbf{Q}=\mathbf{Q}(L, x)=\mathbf{D}(L)+\left(\begin{array}{l}
x \\
0
\end{array}\right),
$$

where formula (6b) can be used for $\mathbf{D}(t)$.

Lemma 3. If $K_{\mathbf{P}}=0$ and $\mathbf{Q}$ is in $\Gamma_{\mathrm{A}}$, a unique clothoid spline spiral of type $\mathrm{A}$ which can join $\mathbf{P}$ to $\mathbf{Q}$ with a Hermite matching exists.

Proof. Let $\mathbf{Q}=\left(\begin{array}{l}Q_{1} \\ Q_{2}\end{array}\right)$; then the conditions that $\mathbf{Q}$ is in $\Gamma_{\mathrm{A}}$ are

$$
Q_{1}>\frac{\pi}{K_{\mathbf{Q}}} C_{I}(\sqrt{W-L})+\frac{1}{K_{\mathbf{Q}}} \sin \frac{\pi}{2} W, \quad 0<L<W,
$$




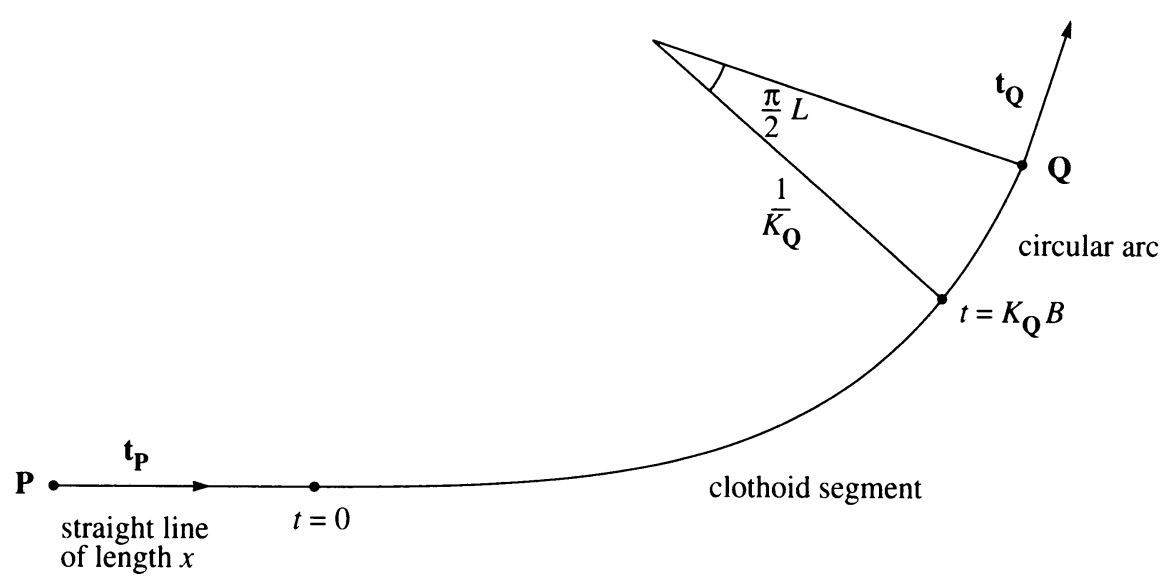

Figure 7. Clothoid spline spiral of type $\mathrm{A}, K_{\mathbf{P}}=0$

and

$$
\frac{1}{K_{\mathbf{Q}}}\left(1-\cos \frac{\pi}{2} W\right)<Q_{2}<\frac{1}{K_{\mathbf{Q}}}\left(1-\cos \frac{\pi}{2} W\right)+\frac{\pi}{K_{\mathbf{Q}}} S_{I}(\sqrt{W}) .
$$

From the given data, the center of the circular arc is

$$
\mathbf{Q}+\frac{1}{K_{\mathbf{Q}}}\left(\begin{array}{c}
-\sin \frac{\pi}{2} W \\
\cos \frac{\pi}{2} W
\end{array}\right) \text {. }
$$

According to [9], a unique clothoid segment joining a line to a circle exists if the distance from the line to the circle is greater than the radius of the circle. The distance from the center of the circle to the $X$-axis is $Q_{2}+\frac{1}{K_{Q}} \cos \frac{\pi}{2} W$, and this is greater than the radius of the circle, $\frac{1}{K_{Q}}$, by the left part of the above condition on $Q_{2}$ that $\mathbf{Q}$ is in $\Gamma_{\mathrm{A}}$. The center of the circle of curvature of the clothoid at $t=K_{\mathbf{Q}} B$ is the same as the center of the circular arc. Comparing the $Y$-coordinates, one obtains

$$
\frac{\pi}{K_{\mathbf{Q}}} S_{I}(\sqrt{W-L})+\frac{1}{K_{\mathbf{Q}}}=Q_{2}+\frac{1}{K_{\mathbf{Q}}} \cos \frac{\pi}{2} W .
$$

The restricted values for $Q_{2}$ and the fact that $S_{I}(t)$ is a strictly monotone increasing function mean that there is a unique solution of that equation satisfying $0<L<W$. Comparing the $X$-coordinates, one gets

$$
x+\frac{\pi}{K_{\mathbf{Q}}} C_{I}(\sqrt{W-L})=Q_{1}-\frac{1}{K_{\mathbf{Q}}} \sin \frac{\pi}{2} W .
$$

The restriction on $Q_{1}$ means that $x$ will be positive. Thus, for any $\mathbf{Q}$ in $\Gamma_{\mathrm{A}}$, there is a unique solution to $\mathbf{Q}=\mathbf{Q}(L, x)$, equation (11), with $0<L<W$ and $x>0$.

Algorithm 2. How to find a clothoid spline spiral of type A with $K_{\mathbf{P}}=0$.

The clothoid spline spiral of type A with $K_{\mathbf{P}}=0$ can be found by solving the nonlinear equation (12) for $L$ by a numerical method, and then solving the linear equation (13) for $x$.

With $K_{\mathbf{P}}>0$, a clothoid spline spiral of type A consists of a circular arc followed by a clothoid segment which is followed by another circular arc 


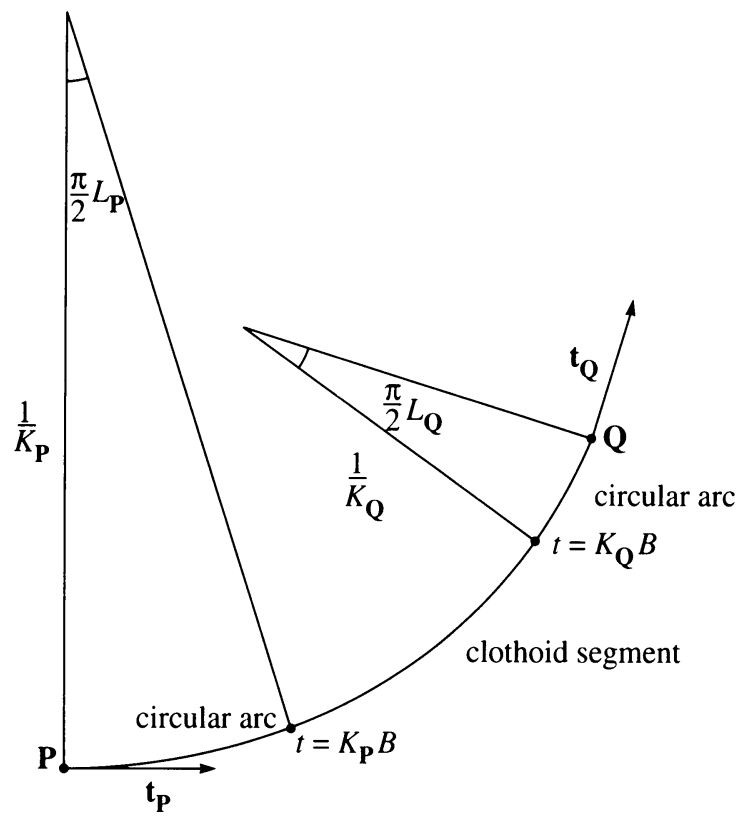

FIGURE 8. Clothoid spline spiral of type $\mathrm{A}, K_{\mathbf{P}}>0$

(see Figure 8). Let the angle of the first circular arc be $\frac{\pi}{2} L_{\mathbf{P}}$, let the scaling factor of the clothoid segment be $\pi B$, and let the angle of the second circular arc be $\frac{\pi}{2} L_{\mathbf{Q}}$. To match curvatures, the parameter in the clothoid segment must run from $t=K_{\mathbf{P}} B$ to $t=K_{\mathbf{Q}} B$. The total angle through which the tangent rotates when traversing the curve from $\mathbf{P}$ to $\mathbf{Q}, \frac{\pi}{2} W$, must equal the sum of the angles through which the tangent rotates over the clothoid segment and over the circular arcs. Equating angles gives

$$
W=L_{\mathbf{P}}+\left(K_{\mathbf{Q}}^{2}-K_{\mathbf{P}}^{2}\right) B^{2}+L_{\mathbf{Q}},
$$

from which $B$ is found to be

$$
B=\sqrt{\frac{W-L_{\mathbf{P}}-L_{\mathbf{Q}}}{K_{\mathbf{Q}}^{2}-K_{\mathbf{P}}^{2}}} .
$$

A formula for $\mathbf{Q}$ is the sum of vectors which join the starting point to the ending point in the first circular arc, the starting point to the ending point in the clothoid, and the starting point to the ending point in the second circular arc:

$$
\begin{aligned}
\mathbf{Q}\left(L_{\mathbf{P}}, L_{\mathbf{Q}}\right)= & \frac{1}{K_{\mathbf{P}}}\left(\begin{array}{c}
\sin \frac{\pi}{2} L_{\mathbf{P}} \\
1-\cos \frac{\pi}{2} L_{\mathbf{P}}
\end{array}\right) \\
& +\pi B R\left(L_{\mathbf{P}}-K_{\mathbf{P}}^{2} B^{2}\right)\left(\begin{array}{c}
C\left(K_{\mathbf{Q}} B\right)-C\left(K_{\mathbf{P}} B\right) \\
S\left(K_{\mathbf{Q}} B\right)-S\left(K_{\mathbf{P}} B\right)
\end{array}\right) \\
& +\frac{1}{K_{\mathbf{Q}}}\left(\begin{array}{c}
\sin \frac{\pi}{2} W-\sin \frac{\pi}{2}\left(W-L_{\mathbf{Q}}\right) \\
-\cos \frac{\pi}{2} W+\cos \frac{\pi}{2}\left(W-L_{\mathbf{Q}}\right)
\end{array}\right),
\end{aligned}
$$

where $R$ is defined in (4). 
The following lemma is used in proving that there is a unique clothoid spline spiral of type A joining $\mathbf{P}$ to $\mathbf{Q}$ with a Hermite matching when $\mathbf{Q}$ is in $\Gamma_{\mathrm{A}}$.

Lemma 4. The Jacobian of $\mathbf{Q}\left(L_{\mathbf{P}}, L_{\mathbf{Q}}\right)$, (14), (15), is nonzero for $0<L_{\mathbf{P}}$, $0<L_{\mathbf{Q}}$, and $L_{\mathbf{P}}+L_{\mathbf{Q}}<W<2$.

Proof. We write (15) in terms of integrals,

$$
\begin{aligned}
\mathbf{Q}\left(L_{\mathbf{P}}, L_{\mathbf{Q}}\right)= & \pi B R\left(L_{\mathbf{P}}-K_{\mathbf{P}}^{2} B^{2}\right) \int_{K_{\mathbf{P}} B}^{K_{\mathbf{Q}} B}\left(\begin{array}{c}
\cos \frac{\pi}{2} u^{2} \\
\sin \frac{\pi}{2} u^{2}
\end{array}\right) d u \\
& +\frac{1}{K_{\mathbf{P}}}\left(\begin{array}{c}
\sin \frac{\pi}{2} L_{\mathbf{P}} \\
1-\cos \frac{\pi}{2} L_{\mathbf{P}}
\end{array}\right)+\frac{1}{K_{\mathbf{Q}}}\left(\begin{array}{c}
\sin \frac{\pi}{2} W-\sin \frac{\pi}{2}\left(W-L_{\mathbf{Q}}\right) \\
-\cos \frac{\pi}{2} W+\cos \frac{\pi}{2}\left(W-L_{\mathbf{Q}}\right)
\end{array}\right) .
\end{aligned}
$$

Bringing the rotation matrix into the integral and making the substitution $U=$ $u^{2}+L_{\mathbf{P}}-K_{\mathrm{P}}^{2} B^{2}$, we can write the integral in the above as

$$
\frac{\pi}{2} \int_{L_{\mathbf{P}}}^{W-L_{\mathbf{Q}}} \frac{B}{\sqrt{U+K_{\mathbf{P}}^{2} B^{2}-L_{\mathbf{P}}}}\left(\begin{array}{c}
\cos \frac{\pi}{2} U \\
\sin \frac{\pi}{2} U
\end{array}\right) d U .
$$

Differentiating $\mathbf{Q}\left(L_{\mathbf{P}}, L_{\mathbf{Q}}\right)$ with respect to $L_{\mathbf{P}}$ and with respect to $L_{\mathbf{Q}}$ yields

$$
\mathbf{Q}_{L_{\mathbf{P}}}\left(L_{\mathbf{P}}, L_{\mathbf{Q}}\right)=\frac{-\pi}{4\left(K_{\mathbf{Q}}^{2}-K_{\mathbf{P}}^{2}\right) B} \int_{L_{\mathbf{P}}}^{W-L_{\mathbf{Q}}} \frac{u-W+L_{\mathbf{Q}}}{\left(u+K_{\mathbf{P}}^{2} B^{2}-L_{\mathbf{P}}\right)^{3 / 2}}\left(\begin{array}{c}
\cos \frac{\pi}{2} u \\
\sin \frac{\pi}{2} u
\end{array}\right) d u
$$

and

$$
\mathbf{Q}_{L_{\mathbf{Q}}}\left(L_{\mathbf{P}}, L_{\mathbf{Q}}\right)=\frac{-\pi}{4\left(K_{\mathbf{Q}}^{2}-K_{\mathbf{P}}^{2}\right) B} \int_{L_{\mathbf{P}}}^{W-L_{\mathbf{Q}}} \frac{u-L_{\mathbf{P}}}{\left.u+K_{\mathbf{P}}^{2} B^{2}-L_{\mathbf{P}}\right)^{3 / 2}}\left(\begin{array}{c}
\cos \frac{\pi}{2} u \\
\sin \frac{\pi}{2} u
\end{array}\right) d u .
$$

The Jacobian of $\mathbf{Q}\left(L_{\mathbf{P}}, L_{\mathbf{Q}}\right)$, $\operatorname{det}\left(\mathbf{Q}_{L_{\mathbf{P}}}, \mathbf{Q}_{L_{\mathbf{Q}}}\right)$, is a positive multiple of

$$
\int_{L_{\mathbf{P}}}^{W-L_{\mathbf{Q}}} \int_{L_{\mathbf{P}}}^{W-L_{\mathbf{Q}}} \frac{u-W+L_{\mathbf{Q}}}{\left(u+K_{\mathbf{P}}^{2} B^{2}-L_{\mathbf{P}}\right)^{3 / 2}} \frac{v \dot{-} L_{\mathbf{P}}}{\left(v+K_{\mathbf{P}}^{2} B^{2}-L_{\mathbf{P}}\right)^{3 / 2}} \sin \frac{\pi}{2}(v-u) d u d v .
$$

The identity (10) transforms the above double integral into

$$
-\int_{L_{\mathbf{P}}}^{W-L_{\mathbf{Q}}} \int_{L_{\mathbf{P}}}^{v} \frac{(v-u)\left(W-L_{\mathbf{P}}-L_{\mathbf{Q}}\right)}{\left(u+K_{\mathbf{P}}^{2} B^{2}-L_{\mathbf{P}}\right)^{3 / 2}\left(v+K_{\mathbf{P}}^{2} B^{2}-L_{\mathbf{P}}\right)^{3 / 2}} \sin \frac{\pi}{2}(v-u) d u d v,
$$

which is negative for $0<L_{\mathbf{P}}, 0<L_{\mathbf{Q}}$, and $L_{\mathbf{P}}+L_{\mathbf{Q}}<W<2$.

Lemma 5. If $K_{\mathbf{P}}>0$ and $\mathbf{Q}$ is in $\Gamma_{\mathrm{A}}$, a unique clothoid spline spiral of type $\mathrm{A}$ which can join $\mathbf{P}$ to $\mathbf{Q}$ with a Hermite matching exists.

Proof. A unique clothoid spline spiral of type A which joins $\mathbf{P}$ to $\mathbf{Q}$ with a Hermite matching exists if there is a unique solution to the equation $\mathbf{Q}=$ $\mathbf{Q}\left(L_{\mathbf{P}}, L_{\mathbf{Q}}\right)$ in (15). A result by Goursat [5, p. 321] shows that a planar mapping function which maps the boundary of a domain to the boundary of the image in a one-to-one manner, and has a nonzero Jacobian over the domain is a oneto-one mapping between the domain and the image. Lemma 4 in conjunction with this result implies that, for any $\mathbf{Q}$ in $\Gamma_{\mathrm{A}}$, there is a unique solution to $\mathbf{Q}=\mathbf{Q}\left(L_{\mathbf{P}}, L_{\mathbf{Q}}\right)$ in (15) with $L_{\mathbf{P}}>0, L_{\mathbf{Q}}>0, L_{\mathbf{P}}+L_{\mathbf{Q}}<W$.

Algorithm 3. How to find a clothoid spline spiral of type A with $K_{\mathbf{P}}>0$. 
The following method for finding the $L_{\mathbf{P}}$ and $L_{\mathbf{Q}}$ is given in [9]. The square of the distance between the centers of the circular arcs is

$$
\left(Q_{1}-\frac{1}{K_{\mathbf{Q}}} \sin \frac{\pi}{2} W\right)^{2}+\left(Q_{2}-\frac{1}{K_{\mathbf{P}}}+\frac{1}{K_{\mathbf{Q}}} \cos \frac{\pi}{2} W\right)^{2} .
$$

The square of the distance between the centers of circles of curvature at $t=K_{\mathrm{P}} B$ and $t=K_{\mathbf{Q}} B$ of a clothoid of scaling factor $\pi B$ is

$$
\left(\frac{\pi C_{I}\left(K_{\mathbf{Q}} B\right)}{K_{\mathbf{Q}}}-\frac{\pi C_{I}\left(K_{\mathbf{P}} B\right)}{K_{\mathbf{P}}}\right)^{2}+\left(\frac{\pi S_{I}\left(K_{\mathbf{Q}} B\right)+1}{K_{\mathbf{Q}}}-\frac{\pi S_{I}\left(K_{\mathbf{P}} B\right)+1}{K_{\mathbf{P}}}\right)^{2} .
$$

These two distances must be equal, and this gives one equation in one unknown, $B$. In [9] it is shown that this equation has a unique solution when the distance between the centers of the circles is less than $1 / K_{\mathbf{P}}-1 / K_{\mathbf{Q}}$ and $B \leq \sqrt{2 /\left(K_{\mathbf{Q}}^{2}-K_{\mathbf{P}}^{2}\right)}$. The first condition is satisfied because $\mathbf{Q}$ is in $\Gamma$, while the second follows from (14) and the restriction that $L_{\mathbf{P}}$ and $L_{\mathbf{Q}}$ are positive with $W<2$. Once $B$ is found by a numerical method, $L_{\mathbf{P}}+L_{\mathbf{Q}}$ can be calculated directly from (14). Looking at the vector joining the centers of the circular arcs, and knowing that the clothoid and the vector joining the centers of its circles of curvature is rotated by $\frac{\pi}{2}\left(L_{\mathbf{P}}-K_{\mathbf{P}}^{2} B^{2}\right)$, it is possible to find $L_{\mathbf{P}}$. Now $L_{\mathbf{Q}}$ can be calculated since the sum $L_{\mathbf{P}}+L_{\mathbf{Q}}$ is known.

\section{Clothoid SPLine SPIRAL OF TYPE B}

In this section, a clothoid spline spiral which can reach any point in $\Gamma_{B}$ will be described. The proof that the clothoid spline spiral is unique for any point in $\Gamma_{B}$, and a short description of an algorithm for finding the clothoid spline spiral, will be given.

A clothoid spline spiral of type B is a clothoid segment followed by a circular arc which is followed by a continuation of the same clothoid (see Figure 9). Let the scaling factor of both clothoid segments be $\pi B$, let the curvature of the circular arc be $K$, and let the angle of the circular arc be $\frac{\pi}{2} L$. To match

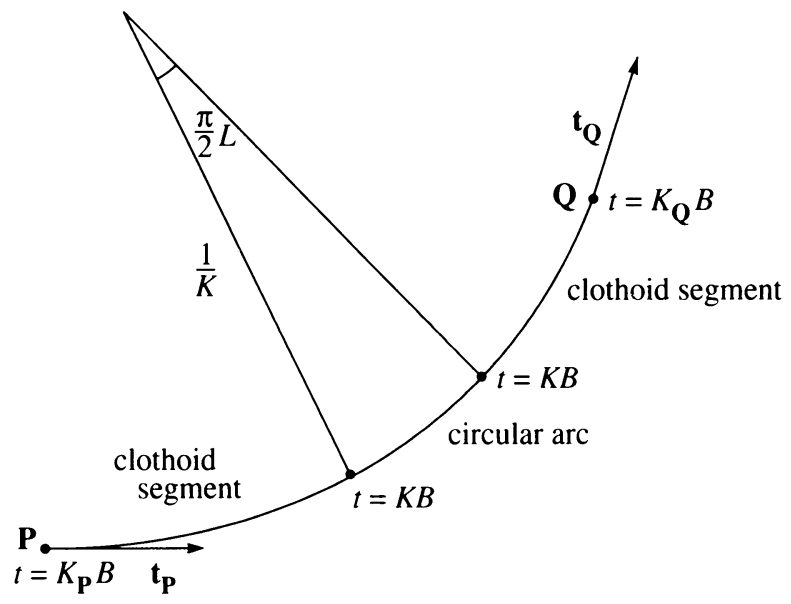

FIGURE 9. Clothoid spline spiral of type B 
curvatures, the parameter in the first clothoid segment must run from $t=K_{\mathbf{P}} B$ to $t=K B$, while the parameter of the second clothoid segment must run from $t=K B$ to $t=K_{\mathbf{Q}} B$. The angle through which the tangent rotates from $\mathbf{P}$ to $\mathbf{Q}, \frac{\pi}{2} W$, must equal the sum of the angles through which it rotates over the two clothoid segments and in the circular arc. Equating angles gives

$$
W=\left(K^{2}-K_{\mathbf{P}}^{2}\right) B^{2}+L+\left(K_{\mathbf{Q}}^{2}-K^{2}\right) B^{2},
$$

from which $B$ is found to be

$$
B=\sqrt{\frac{W-L}{K_{\mathbf{Q}}^{2}-K_{\mathbf{P}}^{2}}} .
$$

A formula for $\mathbf{Q}$ is the sum of the vectors which join the point on the clothoid at $t=K_{\mathrm{P}} B$ to the center of the circular arc, and the center of the circular arc to the point on the clothoid at $t=K_{\mathrm{Q}} B$ :

$$
\begin{aligned}
\mathbf{Q}(K, L)= & R\left(-K_{\mathbf{P}}^{2} B^{2}\right)\left\{\frac{\pi}{K}\left(\begin{array}{c}
C_{I}(K B) \\
S_{I}(K B)+\frac{1}{\pi}
\end{array}\right)-\pi B\left(\begin{array}{c}
C\left(K_{\mathbf{P}} B\right) \\
S\left(K_{\mathbf{P}} B\right)
\end{array}\right)\right\} \\
& +R\left(W-K_{\mathbf{Q}}^{2} B^{2}\right)\left\{\pi B\left(\begin{array}{c}
C\left(K_{\mathbf{Q}} B\right) \\
S\left(K_{\mathbf{Q}} B\right)
\end{array}\right)-\frac{\pi}{K}\left(\begin{array}{c}
C_{I}(K B) \\
S_{I}(K B)+\frac{1}{\pi}
\end{array}\right)\right\},
\end{aligned}
$$

where $R$ is defined in (4).

The following lemma is used in proving that there is a unique clothoid spline spiral of type $\mathbf{B}$ joining $\mathbf{P}$ to $\mathbf{Q}$ with a Hermite matching when $\mathbf{Q}$ is in $\Gamma_{\mathbf{B}}$.

Lemma 6. The Jacobian of $\mathbf{Q}(K, L)$, (16), (17), is nonzero for $K_{\mathbf{P}}<K<K_{\mathbf{Q}}$ and $0<L<W<2$.

Proof. Using the identities (1) to eliminate the integrals of Fresnel integrals, and writing (17) in terms of integrals, one obtains

$$
\begin{aligned}
\mathbf{Q}(K, L)= & \pi B R\left(-K_{\mathbf{P}}^{2} B^{2}\right) \int_{K_{\mathbf{P}} B}^{K B}\left(\begin{array}{l}
\cos \frac{\pi}{2} u^{2} \\
\sin \frac{\pi}{2} u^{2}
\end{array}\right) d u \\
& +\pi B R\left(W-K_{\mathbf{Q}}^{2} B^{2}\right) \int_{K_{\mathbf{Q}} B}^{K B}\left(\begin{array}{l}
\cos \frac{\pi}{2} u^{2} \\
\sin \frac{\pi}{2} u^{2}
\end{array}\right) d u \\
& -\frac{1}{K}\left(\begin{array}{c}
\sin \frac{\pi}{2}\left(K^{2}-K_{\mathbf{P}}^{2}\right) B^{2} \\
-\cos \frac{\pi}{2}\left(K^{2}-K_{\mathbf{P}}^{2}\right) B^{2}
\end{array}\right) \\
& +\frac{1}{K}\left(\begin{array}{c}
\sin \frac{\pi}{2}\left(W-\left(K_{\mathbf{Q}}^{2}-K^{2}\right) B^{2}\right) \\
-\cos \frac{\pi}{2}\left(W-\left(K_{\mathbf{Q}}^{2}-K^{2}\right) B^{2}\right)
\end{array}\right) .
\end{aligned}
$$

Bringing the rotation matrices into the integrals and making the substitution $U=u^{2}-K_{\mathbf{P}}^{2} B^{2}$ in the first integral and $U=K_{\mathbf{Q}}^{2} B^{2}-u^{2}$ in the second, we can write the integrals as

$$
\begin{aligned}
& \frac{\pi}{2} \int_{0}^{\left(K^{2}-K_{\mathbf{P}}^{2}\right) B^{2}} \frac{B}{\sqrt{U+K_{\mathbf{P}}^{2} B^{2}}}\left(\begin{array}{c}
\cos \frac{\pi}{2} U \\
\sin \frac{\pi}{2} U
\end{array}\right) d U \\
& \quad+\frac{\pi}{2} \int_{0}^{\left(K_{\mathbf{Q}}^{2}-K^{2}\right) B^{2}} \frac{B}{\sqrt{K_{\mathbf{Q}}^{2} B^{2}-U}}\left(\begin{array}{l}
\cos \frac{\pi}{2}(W-U) \\
\sin \frac{\pi}{2}(W-U)
\end{array}\right) d U .
\end{aligned}
$$


Differentiating $\mathbf{Q}(K, L)$ of (17) with respect to $K$ and with respect to $L$ yields

$$
\mathbf{Q}_{K}(K, L)=-\frac{2}{K^{2}} \sin \frac{\pi}{4} L\left(\begin{array}{c}
\cos \frac{\pi}{2}\left(\left(K^{2}-K_{\mathbf{P}}^{2}\right) B^{2}+\frac{L}{2}\right) \\
\sin \frac{\pi}{2}\left(\left(K^{2}-K_{\mathbf{P}}^{2}\right) B^{2}+\frac{L}{2}\right)
\end{array}\right)
$$

and

$$
\begin{aligned}
\mathbf{Q}_{L}(K, L)= & \frac{-\pi}{4\left(K_{\mathbf{Q}}^{2}-K_{\mathbf{P}}^{2}\right) B} \int_{0}^{\left(K^{2}-K_{\mathbf{P}}^{2}\right) B^{2}} \frac{u}{\left(u+K_{\mathbf{P}}^{2} B^{2}\right)^{3 / 2}}\left(\begin{array}{c}
\cos \frac{\pi}{2} u \\
\sin \frac{\pi}{2} u
\end{array}\right) d u \\
& +\frac{\pi}{4\left(K_{\mathbf{Q}}^{2}-K_{\mathbf{P}}^{2}\right) B} \int_{0}^{\left(K_{\mathbf{Q}}^{2}-K^{2}\right) B^{2}} \frac{u}{\left(K_{\mathbf{Q}}^{2} B^{2}-u\right)^{3 / 2}}\left(\begin{array}{c}
\cos \frac{\pi}{2}(W-u) \\
\sin \frac{\pi}{2}(W-u)
\end{array}\right) d u .
\end{aligned}
$$

Now, the Jacobian of $\mathbf{Q}(K, L)$, $\operatorname{det}\left(\mathbf{Q}_{K}, \mathbf{Q}_{L}\right)$, is a negative multiple of

$$
\begin{gathered}
\int_{0}^{\left(K^{2}-K_{\mathbf{P}}^{2}\right) B^{2}} \frac{u}{\left(u+K_{\mathbf{P}}^{2} B^{2}\right)^{3 / 2}} \sin \frac{\pi}{2}\left(\left(K^{2}-K_{\mathbf{P}}^{2}\right) B^{2}+\frac{L}{2}-u\right) d u \\
+\int_{0}^{\left(K_{\mathbf{Q}}^{2}-K^{2}\right) B^{2}} \frac{u}{\left(K_{\mathbf{Q}}^{2} B^{2}-u\right)^{3 / 2}} \sin \frac{\pi}{2}\left(\left(K_{\mathbf{Q}}^{2}-K^{2}\right) B^{2}+\frac{L}{2}-u\right) d u .
\end{gathered}
$$

The argument of sine in the first integral varies from $\frac{\pi}{2}\left(\left(K^{2}-K_{\mathrm{P}}^{2}\right) B^{2}+\frac{L}{2}\right)$ to $\frac{\pi L}{4}$. The first quantity is less than

$$
\frac{\pi}{2}\left(\left(K_{\mathbf{Q}}^{2}-K_{\mathbf{P}}^{2}\right) B^{2}+\frac{L}{2}\right)=\frac{\pi}{2}\left(W-L+\frac{L}{2}\right) \leq \pi .
$$

Thus, the sine is always positive in the first integral. The argument of sine in the second integral varies from $\frac{\pi}{2}\left(\left(K_{\mathrm{Q}}^{2}-K^{2}\right) B^{2}+\frac{L}{2}\right)$ to $\frac{\pi L}{4}$. The first quantity is less than $\pi$, so the sine is always positive in the second integral. The Jacobian is nonzero when $K_{\mathbf{P}}<K<K_{\mathbf{Q}}$ and $0<L<W<2$.

Lemma 7. If $\mathbf{Q}$ is in $\Gamma_{\mathrm{B}}$, a unique clothoid spline spiral of type $\mathbf{B}$ which can join $\mathbf{P}$ to $\mathbf{Q}$ with a Hermite matching exists.

Proof. Lemma 6 in conjunction with the result of Goursat [5, p. 321] implies that, for any $\mathbf{Q}$ in $\Gamma_{\mathrm{B}}$, there is a unique solution to $\mathbf{Q}=\mathbf{Q}(K, L)$ in (17) with $K_{\mathbf{P}}<K<K_{\mathbf{Q}}$ and $0<L<W$. This is just what is needed to show that there is a unique clothoid spline spiral of type $\mathbf{B}$ which joins $\mathbf{P}$ to $\mathbf{Q}$ with a Hermite matching.

An effective algorithm for finding the clothoid spline spiral which passes through a given $\mathbf{Q}$ in $\Gamma_{\mathrm{B}}$ is obtained by considering curves $\mathbf{Q}(K, L),(16)$ and (17), of constant $L$ (see Figure 10). Lemma 8 shows that those curves have positive curvature $K$ varying from $K_{\mathbf{P}}$ to $K_{\mathbf{Q}}$. Note that, as $K$ approaches $K_{\mathbf{Q}}$, the curve $\mathbf{Q}(K, L)$ of constant $L$ approaches $\mathbf{D}(L)$.

Lemma 8. Curves $\mathbf{Q}(K, L),(16)$ and (17), of constant $L$ for $K$ varying from $K_{\mathbf{P}}$ to $K_{\mathbf{Q}}$ have positive curvature and a tangent which rotates through an angle of less than $\pi$.

Proof. The derivative of $\mathbf{Q}(K, L)$ with respect to $K, \mathbf{Q}_{K}(K, L)$, is given in (18), while the second derivative with respect to $K$ is

$$
\mathbf{Q}_{K K}(K, L)=\frac{-2}{K} \mathbf{Q}_{K}(K, L)+\frac{2 \pi B^{2}}{K} \sin \frac{\pi}{4} L\left(\begin{array}{c}
\sin \frac{\pi}{2}\left(\left(K^{2}-K_{\mathbf{P}}^{2}\right) B^{2}+\frac{L}{2}\right) \\
-\cos \frac{\pi}{2}\left(\left(K^{2}-K_{\mathbf{P}}^{2}\right) B^{2}+\frac{L}{2}\right)
\end{array}\right) .
$$




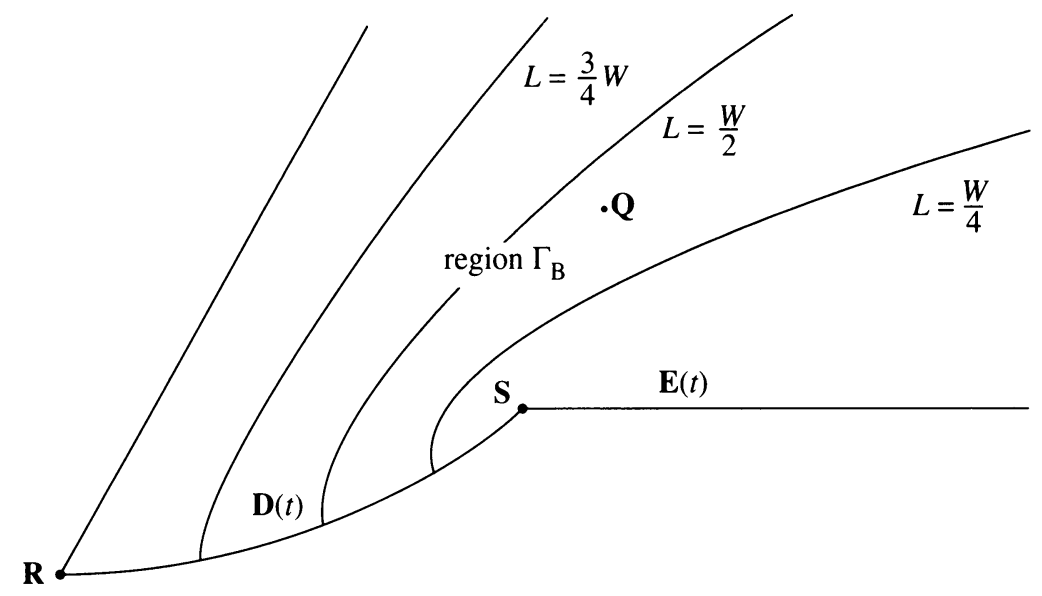

Figure 10. Curves $\mathbf{Q}(K, L)$ of constant $L$ in $\Gamma_{\mathbf{B}}$

The sign of curvature is the same as the sign of $\operatorname{det}\left(\mathbf{Q}_{K}, \mathbf{Q}_{K K}\right)$, and

$$
\operatorname{det}\left(\mathbf{Q}_{K}, \mathbf{Q}_{K K}\right)=\frac{4 \pi B^{2}}{K^{3}} \sin ^{2} \frac{\pi}{4} L,
$$

which is positive; so the curvature of $\mathbf{Q}(K, L), L$ constant, is positive.

The tangent at $K=K_{\mathbf{P}}$ forms an angle of $\pi+\frac{\pi}{4} L$ with the $X$-axis, while the tangent at $K=K_{\mathbf{Q}}$ forms an angle of $\pi+\frac{\pi}{2} W-\frac{\pi}{4} L$ with the $X$-axis. The difference is $\frac{\pi}{2}(W-L)$ which is less than $\pi$ because $W<2$.

Algorithm 4. How to find a clothoid spline spiral of type B.

The algorithm WhichSide [8] can be used to determine whether a given $\mathbf{Q}$ is on a curve of given $L$, on the convex side or on the concave side. If $\mathbf{Q}$ is on the concave side, a smaller $L$ is needed, while, if $\mathbf{Q}$ is on the convex side, a larger $L$ is needed. Since $L$ is restricted to $(0, W)$, a simple bisection algorithm [3] can be used to find the $L$ for which $\mathbf{Q}(K, L)$ passes through $\mathbf{Q}$. The algorithm WhichSide returns both $K$ and $L$ when $\mathbf{Q}$ is on the curve.

If $K_{\mathbf{P}}=0$, the case illustrated in Figure 10, the curves of constant $L$ come from infinity as $K$ increases from zero. The algorithm WhichSide cannot be applied directly because the curve segments are infinite in length. A practical way around this difficulty is to choose a positive lower limit for $K$. Such a restriction on $K$ will put a lower bound on the length of the first clothoid segment. Thus, if $\mathbf{Q}$ is in some parts of $\Gamma_{B}$, the algorithm will not be able to find the required clothoid spline spiral because the first clothoid segment in that clothoid spline spiral is too short.

\section{Conclusion}

Lemmas 3,5, and 7, and an examination of special cases can be combined to give the main mathematical result of the paper.

Theorem 2. If $\mathbf{Q}$ is in $\Gamma$ as defined by the given $W, K_{\mathbf{P}}$, and $K_{\mathbf{Q}}$, then there exists a unique clothoid spline transition spiral which passes through $\mathbf{P}$ and $\mathbf{Q}$, whose tangent at $\mathbf{Q}$ makes an angle of $\frac{\pi}{2} W$ with the $X$-axis, and whose curvature is $K_{\mathbf{P}}$ at $\mathbf{P}$ and $K_{\mathbf{Q}}$ at $\mathbf{Q}$. 
Proof. If $\mathbf{Q}$ is in $\Gamma_{\mathrm{A}}$ and $K_{\mathbf{P}}=0$, the proof is given in Lemma 3 ; if $\mathbf{Q}$ is in $\Gamma_{\mathrm{A}}$ and $K_{\mathbf{P}}>0$, the proof is given in Lemma 5 ; and if $\mathbf{Q}$ is in $\Gamma_{\mathrm{B}}$, the proof is given in Lemma 7.

When $\mathbf{Q}$ is on either of the curves $\mathbf{D}(t)$ or $\mathbf{E}(t)$, the analysis is simple and the results are quoted below. If $\mathbf{Q}$ is on $\mathbf{D}(t), \mathbf{Q}=\mathbf{D}\left(t_{1}\right)$, the clothoid spline spiral which joins $\mathbf{P}$ to $\mathbf{Q}$ is a clothoid segment followed by a circular arc of angle $\frac{\pi}{2} t_{1}$. The tangent to the clothoid rotates $\frac{\pi}{2}\left(K_{\mathbf{Q}}^{2}-K_{\mathbf{P}}^{2}\right) B^{2}$ over the clothoid segment, and the tangent to the clothoid spline spiral rotates $\frac{\pi}{2} W$ over the whole spiral; thus, the scaling factor of the clothoid is

$$
\pi B=\pi \sqrt{\frac{W-t_{1}}{K_{\mathbf{Q}}^{2}-K_{\mathbf{P}}^{2}}} .
$$

If $\mathbf{Q}$ is on $\mathbf{E}(t), \mathbf{Q}=\mathbf{E}\left(t_{2}\right)$, and $K_{\mathbf{P}}=0$, the clothoid spline spiral is a straight line of length $t_{2}$ followed by a clothoid segment. The tangent to the clothoid rotates through an angle of $\frac{\pi}{2} K_{\mathbf{Q}}^{2} B^{2}=\frac{\pi}{2} W$; thus, the scaling factor of the clothoid segment is

$$
\pi B=\pi \frac{\sqrt{W}}{K_{\mathrm{Q}}} .
$$

If $\mathbf{Q}$ is on $\mathbf{E}(t), \mathbf{Q}=\mathbf{E}\left(t_{3}\right)$, and $K_{\mathbf{P}}>0$, the clothoid spline spiral is a circular arc of angle $\frac{\pi}{2} t_{3}$ followed by a clothoid segment. The scaling factor of the clothoid can be calculated as above and is

$$
\pi B=\pi \sqrt{\frac{W-t_{3}}{K_{\mathbf{Q}}^{2}-K_{\mathbf{P}}^{2}}} .
$$

Algorithm 5. How to find the unique clothoid spline spiral to join $\mathbf{P}$ to $\mathbf{Q}$ with a Hermite matching.

The first step, deciding if $\mathbf{Q}$ is in $\Gamma$, is easy as the boundaries of $\Gamma$ are straight lines and circular arcs. Algorithm 1 shows how to decide whether $\mathbf{Q}$ is in $\Gamma_{A}, \Gamma_{B}$ or on the boundary dividing those two regions. If $\mathbf{Q}$ is in $\Gamma_{A}$, Algorithms 2 and 3 show how to find the clothoid spline spiral which joins $P$ to $\mathbf{Q}$. If $\mathbf{Q}$ is in $\Gamma_{\mathrm{B}}$, Algorithm 4 shows how to find the clothoid spline spiral which joins $\mathbf{P}$ to $\mathbf{Q}$. If $\mathbf{Q}$ is on the boundary, the clothoid spline spiral can be found as outlined in the proof of Theorem 2 .

\section{ACKNOWLEDGMENT}

The authors appreciate the comments of an anonymous referee which greatly helped to improve the presentation of this paper.

\section{BIBLIOGRAPHY}

1. M. Abramowitz and I. A. Stegun (eds.), Handbook of mathematical functions, Dover, New York, 1965.

2. K. G. Baass, The use of clothoid templates in highway design, Transportation Forum 1 (1984), 47-52.

3. R. L. Burden and J. D. Faires, Numerical analysis, 3rd ed., Prindle, Weber and Schmidt, Boston, 1985.

4. G. Farin, Curves and surfaces for computer aided geometric design: a practical guide, Academic Press, San Diego, 1988. 
5. E. Goursat, $A$ course in mathematical analysis, Vol. I (E. R. Hedrick, translator), Ginn, Boston, 1904.

6. H. W. Guggenheimer, Differential geometry, McGraw-Hill, New York, 1963.

7. M. A. Heald, Rational approximations for the Fresnel integrals, Math. Comp. 44 (1985), 459-461; Corrigendum, ibid., 46 (1986), 771.

8. D. S. Meek and R. S. D. Thomas, A guided clothoid spline, Comput. Aided Geom. Design 8 (1991), 163-174.

9. D. S. Meek and D. J. Walton, The use of Cornu spirals in drawing planar curves of controlled curvature, J. Comput. Appl. Math. 25 (1989), 69-78.

10. E. Mehlum, Nonlinear splines, in Computer Aided Geometric Design (R. E. Barnhill and R. F. Riesenfield, eds.), Academic Press, New York, 1974, pp. 173-207.

11. E. Mehlum and J. Wimp, Spherical curves and quadratic relationships for special functions, J. Austral. Math. Soc. Ser. B 27 (1985), 111-124.

12. J. Stoer, Curve fitting with clothoidal splines, J. Res. Nat. Bur. Standards 87 (1982), 317-346.

13. D. J. Walton and D. S. Meek, Computer aided design for horizontal alignment, ASCE J. Transportation Engrg. 115 (1989), 411-424.

Department of Computer Science, University of Manitoba, Winnipeg, Manitoba R3T 2N2, CANADA

E-mail address, D. S. Meek: dmeek@ccu.umanitoba.ca

E-mail address, D. J. Walton: dwalton@ccm.umanitoba.ca 